\title{
Geometry Effect on the Strain-Induced Self-Rolling of Semiconductor Membranes
}

\author{
Ik Su Chun, ${ }^{\dagger, \|}$ Archana Challa, ${ }^{\dagger, \|}$ Brad Derickson, ${ }^{\ddagger}$ K. Jimmy Hsia, ${ }^{*},, \S$ and Xiuling Li ${ }^{*}, \uparrow, \S$ \\ ${ }^{\dagger}$ Department of Electrical and Computer Engineering, ${ }^{\star}$ Department of Mechanical Science and Engineering, and \\ ${ }^{\S}$ Micro and Nanotechnology Laboratory, University of Illinois, Urbana, Illinois 61801
}

\begin{abstract}
Semiconductor micro- and nanotubes can be formed by strain-induced self-rolling of membranes. The effect of geometrical dimensions on the self-rolling behavior of epitaxial mismatch-strained $\operatorname{In}_{x} \mathrm{Ga}_{1-x} \mathrm{As}-\mathrm{GaAs}$ membranes are systematically studied both experimentally and theoretically using the finite element method. The final rolling direction depends on the length and width of the membrane as well as the diameter of the rolled-up tube. The energetics of the final states, the history of rolling process, and the kinetic control of the etching anisotropy ultimately determine the rolling behavior. Results reported here provide critical information for precise positioning and uniform large area assembly of semiconducting micro- and nanotubes for applications in photonics, microelectromechanical systems, etc.
\end{abstract}

KEYWORDS Strain-induced self-rolling, semiconductor micro- and nanotubes, GaAs MOCVD, finite element method

A s reported recently, semiconductor micro- and nanotubes possess potentials to provide a wide range of functionalities for optoelectronics, plasmonics, microelectromechanical systems (MEMS), and sensing. ${ }^{1-6}$ These tubes are formed by self-rolling of strained thin-film membranes that are epitaxially grown and lithographically defined. ${ }^{7}$ To achieve desirable structure and thus functionalities of this type of tubular building blocks, understanding the mechanisms of rolling and parameters controlling the rolling process is therefore critical. In contrast to the common bottom-up self-assembly techniques (e.g., self-assembled quantum dots), the formation method for rolled-up tubes here combines the bottom-up aspect of epitaxial growth with the top-down side of lithography patterning. This approach makes feasible the fabrication of large arrays and integration with existing semiconductor processing technology, while maintaining the control of tube size and heterojunction formation in the tube wall. Recently, we have reported the large area assembly, dispersion, and transfer-printing of ordered InGaAs/GaAs nanotube arrays using such straindriven self-rolling mechanism. ${ }^{6,8}$ In this article, we systematically address yet another parameter important for the formation of ordered large arrays of self-rolled-up tubes, the geometry effect of the membrane on tube length and orientation.

It is well-established that mismatch strain between different layers in thin film systems can induce mechanical deformation either in the form of surface waviness formation $^{9-12}$ or in the form of bending and rolling of thin membranes. ${ }^{13,14}$ When two mismatched epitaxial layers are

*To whom correspondence should be addressed, xiuling@illinois.edu and kjhsia@illinois.edu.

"These authors contributed to the paper equally.

Received for review: 05/11/2010

Published on Web: 00/00/0000 constrained to a substrate, a tremendous amount of residual strain energy is stored in the bilayer membrane. Once it is released from the constraint of the substrate, the membrane relieves the stored strain energy by rolling up to form various 3D structures including micro- and nanotubes. The tube diameter is mostly determined by the total layer thickness and the level of mismatch strain (determined by the composition) in the epitaxial layers. The self-rolling direction is influenced by the elastic anisotropy in $\mathrm{A}^{3} \mathrm{~B}^{5}$ cubic crystals. ${ }^{7,8,15}$ For $\operatorname{In}_{x} \mathrm{Ga}_{1-x}$ As-GaAs materials, the crystal is less stiff along $\langle 100\rangle$ directions than along $\langle 110\rangle$ directions. ${ }^{16}$ Taking advantage of the crystal orientation dependence and geometric aspect ratio, various shapes including helices, spirals, tubes, rings, etc., 4,17,18 can be formed using the self-rolling mechanism by tailoring the shape and orientation of the thin membrane relative to the $\langle 100\rangle$ "soft" direction. Huang et al. has also developed a theoretical model to predict the formation of nanorings, nanodrills, and nanocoils as a function of the geometry of the strained bilayer films. ${ }^{18}$ When the sides of a rectangular-shaped membrane are orientated along the soft rolling direction, all four sides are crystalgraphically equivalent. As the membrane is gradually released off the substrate through lateral etching of the sacrificial layer, all four sides should have equal tendency to roll up. However, as will be demonstrated in this article, for crystalgraphically equivalent directions, the rolling behavior varies depending on the size and aspect ratio of the starting membrane as well as the built-in strain and thickness of the membrane.

Specifically, we report on systematic experimentation as well as numerical simulation results of the geometry dependence of the self-rolling behavior of $\operatorname{In}_{x} \mathrm{Ga}_{1-x} \mathrm{As}-\mathrm{GaAs}$ semiconductor micro- and nanotubes. A key question we intend to answer is whether the nanotube formation is controlled by energetics alone or also by a history-dependent process 

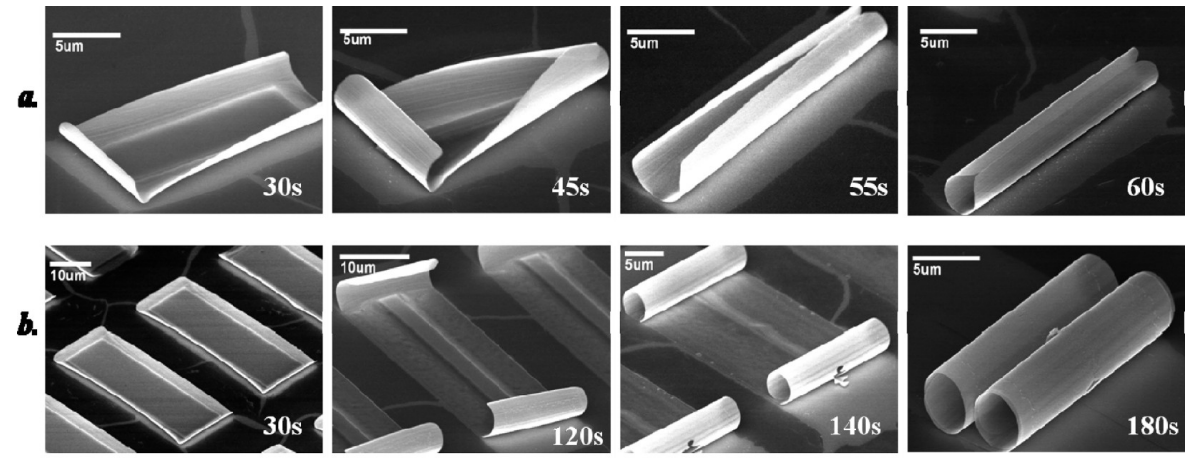

FIGURE 1. Time evolution of membrane rolling from two different directions. Snapshots of SEM images of the rolling process of In ${ }_{x} \mathrm{Ga}_{1-x} \mathrm{As}_{\mathrm{S}}$ GaAs rectangular membranes after $\mathrm{Al}_{0.75} \mathrm{Ga}_{0.25}$ As layer sacrificial layer is etched for the indicated time in $1: 2 \mathrm{HF}: \mathrm{H}_{2} \mathrm{O}$ solution: (a) $10 \times 25 \mu \mathrm{m}$ membrane $\left(\mathrm{In}_{0.3} \mathrm{Ga}_{0.7} \mathrm{As} / \mathrm{GaAs} 38 \mathrm{~nm}\right.$ thick) etched for 30, 45, 55, and $60 \mathrm{~s}$ to form a $3 \mu \mathrm{m}$ diameter tube rolling from the long side; (b) $19 \times$ $50 \mu \mathrm{m}$ membrane $\left(\mathrm{In}_{0.3} \mathrm{Ga}_{0.7} \mathrm{As} / \mathrm{GaAs} 38 \mathrm{~nm}\right.$ thick) etched for $30,120,140$, and $180 \mathrm{~s}$ to form a $4 \mu \mathrm{m}$ diameter $\left(\mathrm{In}_{0.2} \mathrm{Ga}_{0.7} \mathrm{As} / \mathrm{GaAs} 38 \mathrm{~nm}\right.$ thick) tube rolling from the short side.

during rolling. Our findings could shine light on one of the major challenges that the nanotechnology field needs to overcome, i.e., the fabrication of nano-objects with precisely controllable dimensions and position.

Micro- and nanotubes for this study were fabricated from pseudomorphically strained epitaxial films grown by metal organic chemical vapor deposition (MOCVD). The epitaxial structure consists of $\operatorname{In}_{x} \mathrm{Ga}_{1-x} \mathrm{As}(0.15<x<0.33)$, GaAs layers or stacks with thicknesses ranging from 12 to $50 \mathrm{~nm}$, and a sacrificial $\mathrm{Al}_{x} \mathrm{Ga}_{1-x} \mathrm{As}$ layer $(x>0.6)$ on a (100) on-axis GaAs substrate. Tubes of different diameters can be produced by varying the thickness and indium composition, thus the mismatch strain and bending rigidity of the films. Photolithography masks consisting of rectangular patterns with different lengths and widths ranging from 1 to $50 \mu \mathrm{m}$ were used to study the geometry effect on rolling behavior. Wet chemical etching $\left(1: 8: 80=\left(\mathrm{H}_{2} \mathrm{SO}_{4}: \mathrm{H}_{2} \mathrm{O}_{2}: \mathrm{H}_{2} \mathrm{O}\right)\right)$ was carried out to transfer the lithographic pattern into the bilayer. The sacrificial layer was then selectively etched to release the bilayer from the substrate using a $\mathrm{HF}$ solution $\left(=\mathrm{HF}: \mathrm{H}_{2} \mathrm{O}\right)$. Scanning electron microscopy (SEM) imaging was used to characterize the nanotubes.

Finite element method (FEM) was used to simulate the rolling behavior of the nanotubes using ABAQUS. The bilayer of $\operatorname{In}_{x} \mathrm{Ga}_{1-x} \mathrm{As}$ and $\mathrm{GaAs}$ was simulated using eight-node thick-shell elements which permit variation in properties through the thickness. The materials are assumed to be elastic. Parameters for the elastic properties of each material used in the simulation are taken from literature. ${ }^{19,20}$ To simulate the epitaxial mismatch strain between the layers, different in-plane coefficients of thermal expansion were assigned to the $\operatorname{In}_{x} \mathrm{Ga}_{1-x}$ As and GaAs layers (in the simulations, the thermal expansion coefficient of GaAs is assigned to be zero). A mismatch strain of prescribed magnitude can then be induced by introducing a temperature change. To reduce computational time, symmetry condition was invoked, and only one-quarter of the membrane plate was modeled by applying the appropriate boundary conditions.
In all simulations, the temperature change is ramped up until the prescribed mismatch strain level is reached. For a given geometry (length, width, and the dimensions of the etched portion), only one rolling mode is achieved as the temperature change increases. However, to understand the controlling mechanisms of the rolling process, the total strain energy of different rolling behaviors (long side, short side, or mixed rolling) needs to be evaluated. To simulate different rolling behavior, we applied a perturbation to the model. During the initial stage of the simulation, one of the edges would be clamped down to promote rolling of the other edge. A small temperature change was applied, and the edge was then released for the remainder of the simulation. For example, to induce rolling along the short side edge, the long side edge was initially clamped during the perturbation and subsequently released. It was observed that once one side (long or short or mixed) started to roll, it would continue to roll even after the artificial clamping of the other side is released, regardless whether it has a higher total strain energy. The resulting model allowed for energy calculations to be made at a fixed point in the etching process. Various points during the etching process could be simulated by changing the dimensions. By comparison of the energy states associated with the two rolling directions, the most thermodynamically preferred rolling direction could be determined.

Figure 1 shows the experimental time evolution of the rolling processes of two single rectangular $\mathrm{In}_{0.2} \mathrm{Ga}_{0.8} \mathrm{As}-\mathrm{GaAs}$ membranes of different geometries as they are released from the substrate by undercutting the sacrificial layer through selective lateral etching. As etching proceeds isotropically from all sides, the center part of the membrane restricted by the substrate becomes narrower until it is completely free. The etching time is indicated on each snapshot. Figure 1 a shows the evolution of the rolling state of a $10 \mu \mathrm{m} \times 25 \mu \mathrm{m}$ rectangular membrane at four different etching times. After $30 \mathrm{~s}$ etching time, the short side of the rectangle started to roll up on one end while at the other end 

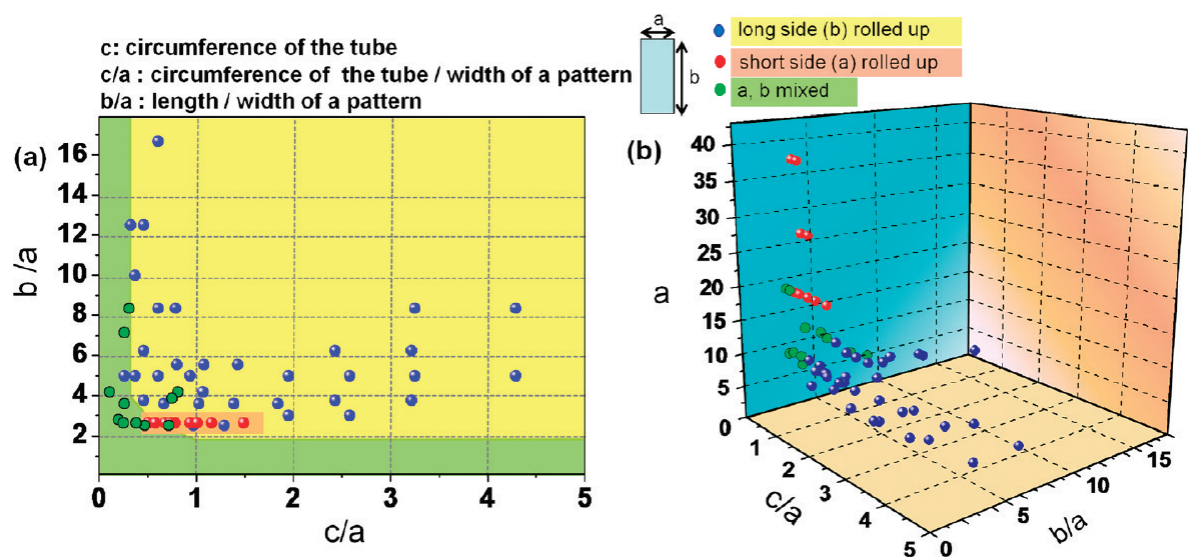

FIGURE 2. Rolling trend versus geometry. (Left) Two-dimensional and (Right) three-dimensional plots summarizing rolling direction as a function of circumference of the tube $(C)$, width $(a)$, and length $(b)$ of the rectangular-shaped membrane. Three types of rolling trends are color coded (blue for long side, red for short side, and green for mixed) on the plot.

the long side started to curve up. At $45 \mathrm{~s}$, the rolling seems to come to a dead-lock since further rolling from either direction is restricted by the other end. At $55 \mathrm{~s}$, however, the long side rolling takes place, implying that the curved short side compromised by opening itself up to allow continued rolling from the long side. A completely rolled-up tube from the long side is formed at $60 \mathrm{~s}$, with the length of the tube equal to the length of the rectangle membrane. The dramatic change in shape between the $45 \mathrm{~s}$ and $55 \mathrm{~s}$ snapshots is a testimony of the mechanical flexibility and compliance for ultrathin inorganic semiconductor membranes, and no extended defects have been found in the rolled-up tubes based to transmission electron microscopy examinations. ${ }^{21}$ This also implies that it is the minimization of the total energy of the system that determines the final rolling shape, even when the initial rolling shape governed by the edge effect is very different.

Membranes of different dimensions have been observed to exhibit different rolling behavior. As shown in Figure $1 \mathrm{~b}$ where a $19 \mu \mathrm{m} \times 50 \mu \mathrm{m}$ rectangular stripe was the starting point, all four sides seemed to curve up equally after $30 \mathrm{~s}$ of etching time. At $120 \mathrm{~s}$, the long side clearly straightened up to yield to the continued rolling of the short side, while the middle of the stripe remains anchored to the substrate. As the undercut etching proceeds, the short sides complete one complete revolution (at $140 \mathrm{~s}$ ) and continues to roll to form multiturn tubes on each end until they met each other in the middle at $180 \mathrm{~s}$. This is in contrast to the final geometry in Figure $1 \mathrm{a}$ where the tube is rolled up along the long side of the rectangle. Note that sometimes small tears occur (e.g., at $140 \mathrm{~s}$ ), especially for cases with multiple turns, which does not seem to stop or change the rolling direction.

Figure 2 summarizes the observed rolling behavior of rectangular geometry membranes systematically varied with length and width in the range of $5-100 \mu \mathrm{m}$ and tube diameter of $0.5-10 \mu \mathrm{m}$, when arrays of identical geometry membranes are released to roll. The rolling direction trend is plotted as a function of three parameters, bla, Cla, and $a$, where $C$ is the circumference of the tube and $a$ and $b$ are the length and width of the rectangular-shaped membrane, respectively. There are three types of rolling behavior. The blue dots represent geometries which result in dominantly long side rolling, red dots represent short side rolling, and green dots are mixed cases with some tubes rolled up from the long side and some from the short side. It is noted that the total strain (lattice mismatch and thickness) in the bilayer solely governs the final diameter of the tube and hence is independent of the length and width of the strip. Accordingly, rolling in either direction does not change the diameter of the resulting tube. Clearly, Figure 2 shows that the rolling direction depends on not only the dimension of the starting membrane $(a$ and $b$ ) but also the tube diameter or circumference (C). The 2D plot shows the rolling behavior in the parameter space of two normalized dimensions ( $\mathrm{Cla}$ and bla). Using a 3D plot where $a$, the width of the rectangle, is added as the third axis, the three types of rolling behavior can be better delineated as a function of geometry. It can be seen that when the tube circumference is much larger than the width $(C / a>2)$, or the aspect ratio of the rectangle is high (b/a > 9), rolling always occurs from the long side (blue dots). When the tube circumference is much smaller than the width $(C / a \ll 1$, i.e., a curved surface but not a complete tube) and aspect ratio of the membrane pattern (b/a) is not very high, the rolling results in a mixed yield (green dots) of long- and short-side rolling, as well as the "dead-locked turnover" shape (see second frame in Figure 1a). For the same aspect ratio (see the 3D figure), long side rolling seems to be limited to smaller membranes, and mixed and short side rolling are more likely to occur for larger ones. Interestingly, for the rectangle dimensions we studied $(1-100 \mu \mathrm{m})$, only a specific ratio of width and length $(b / a \sim 2.6)$ has yielded tubes rolled up from the short side, regardless of diameter (4, 7 , and $9 \mu \mathrm{m}$ shown) and absolute $a$ and $b$ dimensions $\left(19 \times 50,29 \times 75,38 \times 100 \mu \mathrm{m}^{2}\right.$ shown $)$.

To explain the experimental observations and reveal the microscopic rolling mechanism, FEM modeling is carried out 


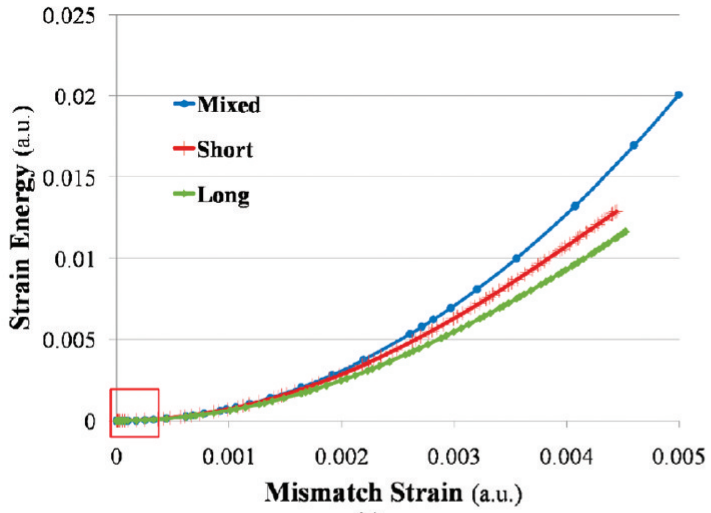

(a)

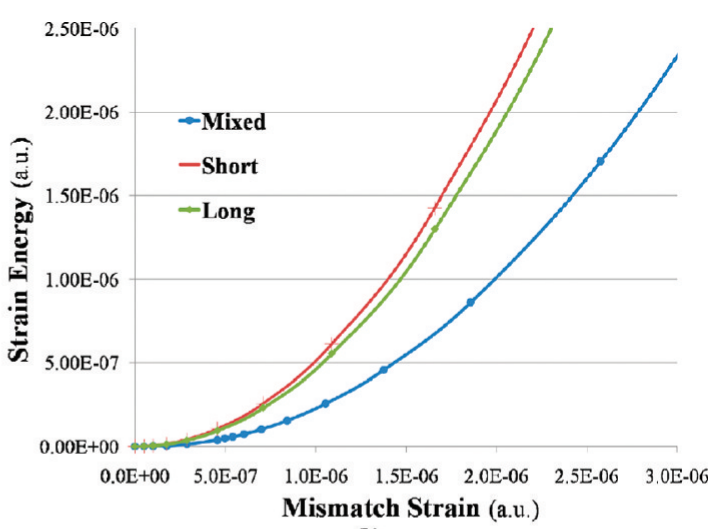

(b)
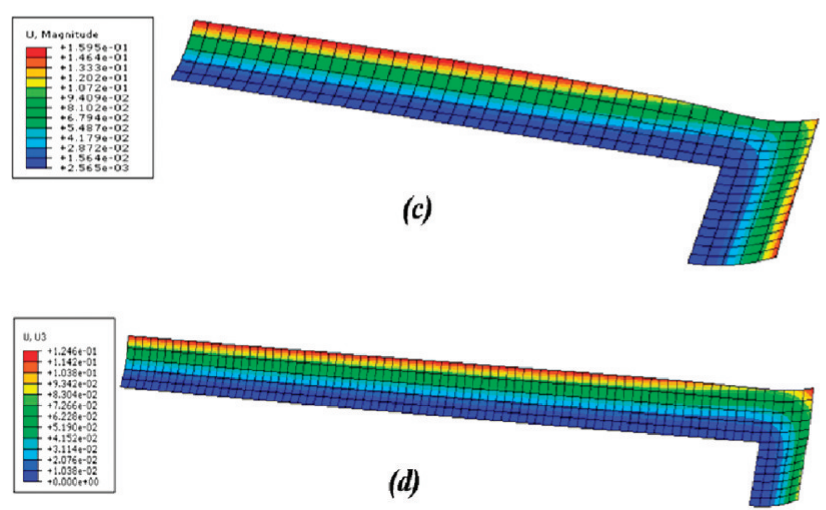

FIGURE 3. FEM simulation results for restricted rolling (analogous to experimental condition). (a) A plot of total strain energy in the three rolling states (long side, short side, and mixed) of a strained membrane $(60 \mu \mathrm{m} \times 100 \mu \mathrm{m})$ as a function of mismatch strain; (b) zoomed-in view of the very early stage of the rolling (red boxed area in (a)); (c) $19 \mu \mathrm{m} \times 50 \mu \mathrm{m}$ and (d) $4 \times 25 \mu \mathrm{m}$ rectangular membrane patterns after $40 \%$ of the total area is released. The colors in the legend indicate extent of displacement starting from highest (red) to lowest displacement from before rolling (blue). (lower right) Schematic showing the geometric parameters of one quadrant of the bilayer membrane used in the FEM model.

to simulate the membrane deformation under analogous conditions. Although self-rolled-up tubes and related architectures have been simulated previously (e.g., refs 14 and 17), the constrained rolling cases with the center region attached to the substrate have not been considered. As will be shown below, It is in fact this constrained rolling that directly influences the rolling direction.

Figure 3 shows the FEM results of a bilayer membrane of $60 \mu \mathrm{m} \times 100 \mu \mathrm{m}$ in dimension under the corresponding experimental condition where the membrane is released from the substrate and deformed gradually through isotropic etching. Parts $\mathrm{a}$ and $\mathrm{b}$ of Figure 3 are plots of the calculated total strain energy of the membrane at a series intermediate rolling states as a function of the "mismatch strain" for three rolling trends (long side, short side, and mixed). It should be pointed out that in the experiments, the constraining geometry of the rolling membrane changes continuously as the film is released from the substrate by isotropic etching. In the FEM simulations, different release states are modeled by changing the dimensions of the unreleased center region $\left(a_{1}\right.$ and $b_{1}$ in Figure 3$)$. If the rolling process is governed by minimizing the total energy stored in the system, there should be a one-to-one correspondence between the experimental process and simulation model. It can be seen from Figure $3 \mathrm{a}$ that, for this particular geometry, the long side rolling is the lowest energy state for the restricted rolling scheme because curving up the long side relaxes more residual strain in the membrane. However, at the very early stage of rolling as shown in Figure 3b, which is the zoomedin view of the red box in Figure 3a, the mixed state where all sides curve up is actually favored energetically, consistent with the experimental observations (Figure 1). It should be pointed out that in all simulations of the isotropic etching cases (i.e., the dimensions of the rolling part along the long side is equal to that along the short side, $a_{0}-a_{1}=b_{0}-b_{1}$ in the quadrant illustration in Figure 3), long side rolling is always energetically preferred.

However, we did observe short-side rolling in membranes with special geometric aspect ratios experimentally (Figure 2). To identify the mechanism for the short-side rolling behavior observed experimentally, we carefully analyzed the time evolution thus intermediate states of film deformation in the FEM simulations. Parts $\mathrm{c}$ and $\mathrm{d}$ of Figure 3 show the simulation results of the deformation profile for two mem- 

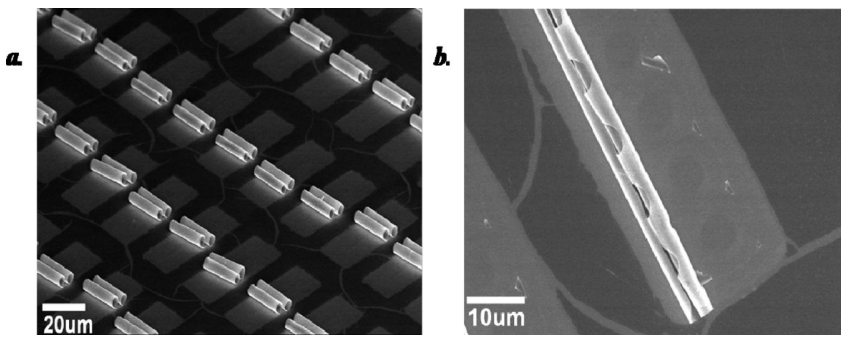

FIGURE 4. Forced rolling. SEM images showing change in rolling behavior from (a) short-side rolling to (b) long-side rolling when holes are lithographically patterned on a $19 \mu \mathrm{m} \times 50 \mu \mathrm{m}$ membrane pattern (38 $\mathrm{nm}$ thick) to induce anisotropic etching and forced rolling along faster etching direction. Etching time in 1:2 HF:DI solution are 180 and $20 \mathrm{~s}$ for (a) and (b), respectively.

brane geometries: (c) $19 \times 50 \mu \mathrm{m}^{2}$ and (d) $4 \times 25 \mu \mathrm{m}^{2}$, when $40 \%$ of the total area is released from the substrate. In Figure $3 \mathrm{c}$, while the edge along the short side is lifted completely, a part of the long-side edge and the corner seem to be pinned down, inferring continued rolling along the short side. In contrast, Figure $3 d$ displays a different behavior in that the corner and the long side are rolled up more than the short side, inferring long-side rolling eventually. These simulations are in agreement with the experimental results which show that while a $19 \times 50 \mu \mathrm{m}^{2}$ strip exhibits shortside rolling, a $4 \times 25 \mu \mathrm{m}^{2}$ strip consistently exhibits longside rolling. Although it is true that the lowest energy configuration of the final state plays an important role in determining the ultimate rolling direction, the simulation and experimental results in the current study imply that the deformation history (which could influence the experimental process such as etching rate in different directions) may also determine the rolling direction depending on the intermediate states. We do not believe that the specific aspect ratio of the $19 \times 50 \mu \mathrm{m}^{2}$ strip for the observed short-side rolling in Figure 2 has significant physical meaning, other than the fact that it seems to lead to an intermediate state that favors short-side rolling.

Our FEM simulations also indicate that, when anisotropic etching is achieved (e.g., when $a_{0}-a_{1}<b_{0}-b_{1}$ ), one can control the rolling behavior and change the rolling direction readily (for details, please see ref 22). Similarly, we can kinetically create an anisotropic etching experimental condition to give rise to asymmetric releasing from each side of the rectangle film and deliberately change the rolling direction. Shown in Figure 4 is one such example where the membrane geometry normally yields short-side rolling (Figure 4a) will roll up along the long side (Figure 4b) upon patterning arrays of etching holes along the long side to induce preferential etching, resulting in long-side rolling. Recently, the use of a U-shaped mesa has been developed to achieve rolled-up quantum dot tubes suspended from the substrate for better optical mode confinement. ${ }^{1,23}$ In this case, the rolling direction is forced by a lithographically defined window for deep etching after shallow etching defines the U-shape mesa. ${ }^{1,23}$

With the systematic mapping and understanding of geometry dependence of the rolling direction, we can readily fabricate perfectly ordered arrays of rolled-up micro- and nanotubes over large areas. Shown in Figure 5 is larger than millimeter size area of such ordered tube arrays with $100 \%$ yield of rolling along the long side.

In summary, we have demonstrated systematically the geometry effect on the strain-driven deformation behavior of self-rolling mismatched epitaxial In $_{x} \mathrm{Ga}_{1-x} \mathrm{As}-\mathrm{GaAs}$ membranes with rectangular geometry both experimentally and using FEM simulation. We have found that the final rolling direction depends on the length and width of the membrane as well as the diameter of the roll-up tubes. The energetics of the final state, the history of rolling process, and the kinetic control of the etching isotropy are all contributing

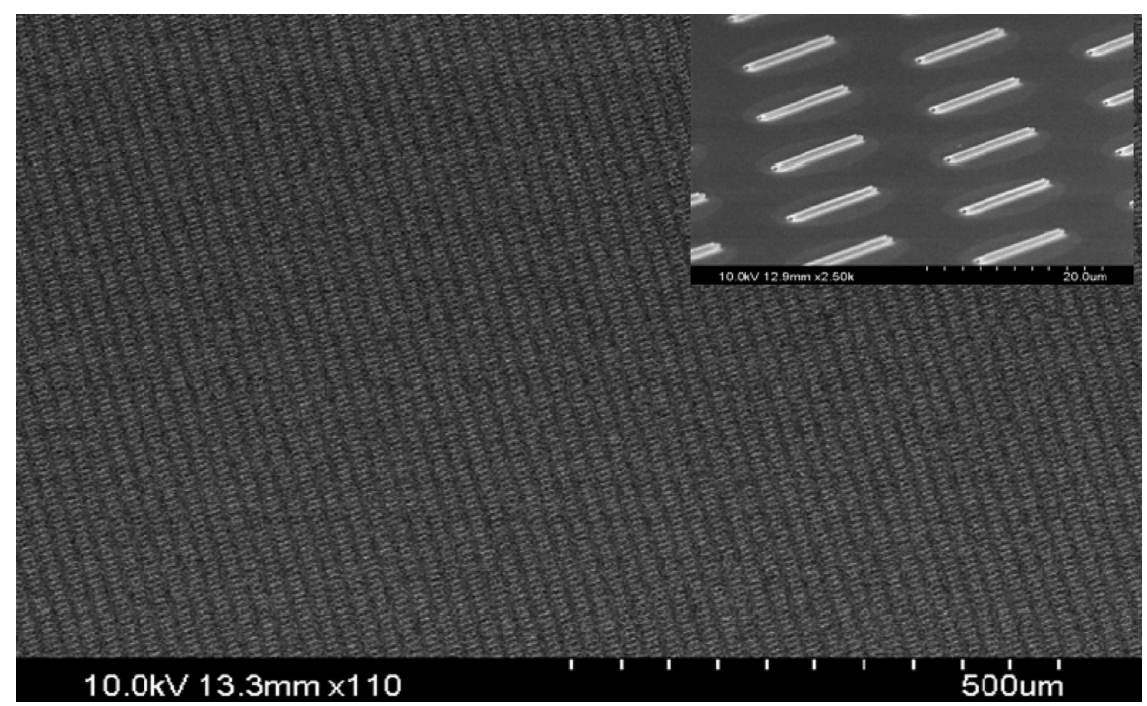

FIGURE 5. Large area assembly. SEM image of a millimeter size area of $\operatorname{In}_{x} \mathrm{Ga}_{1-x} \mathrm{As} / \mathrm{GaAs}(x=0.3)$ tube array that consists of perfectly aligned long side rolled-up $600 \mathrm{~nm}$ diameter microtubes formed from a $14 \mathrm{~nm}$ thick $3 \times 25 \mu \mathrm{m}^{2}$ rectangular membrane mesa. 
factors to the rolling behavior. The revelation of these governing mechanisms as well as the guideline provided in this study should have significant impact on the precise control of tubular and curved semiconductor nanostructures for better applications in MEMS/NEMS, nanoelectronics, and nanophotonics.

Acknowledgment. This work is supported by the National Science Foundation (NSF) awards ECCS 0747178 (X. Li's CAREER award), CMMI 0749028 (X. Li), DMR 0504751 (K. J. Hsia), and CMMI 0952565 (K. J. Hsia).

\section{REFERENCES AND NOTES}

(1) Li, F.; Mi, Z.; Vicknesh, S. Opt. Lett. 2009, 34, 2915-2917.

(2) Strelow, C.; Rehberg, H.; Schultz, C. M.; Welsch, H.; Heyn, C.; Heitmann, D.; Kipp, T. Phys. Rev. Lett. 2008, 101, 127403-4.

(3) Schwaiger, S.; Broll, M.; Krohn, A.; Stemmann, A.; Heyn, C.; Stark, Y.; Stickler, D.; Heitmann, D.; Mendach, S. Phys. Rev. Lett. 2009, 102, 163903-4

(4) Prinz, V. Y.; Seleznev, V. A.; Prinz, A. V.; Kopylov, A. V. Sci. Technol. Adv. Mater. 2009, 10, No. 034502

(5) Huang, G. S.; Mei, Y. F.; Thurmer, D. J.; Coric, E.; Schmidt, O. G. Lab Chip 2009, 9, 263-268

(6) Li, X.J. Phys. D: Appl. Phys. 2008, 41, 193001.

(7) Prinz, V. Y.; Seleznev, V. A.; Gutakovsky, A. K.; Chehovskiy, A. V.; Preobrazhenskii, V. V.; Putyato, M. A.; Gavrilova, T. A. Physica E 2000, 6, 828-831.
(8) Chun I. S.; Li, X. IEEE Trans. Nanotechnol 2008, 7, 493-495.

(9) Khang, D.-Y.; Jiang, H.; Huang, Y.; Rogers, J. A. Science 2006, 311 , 208-212.

(10) Panat, R.; Hsia, K. J.; Cahill, D. G. J. Appl. Phys. 2005, 97, No. 013521-7.

(11) Rahul, P.; Sulin, Z.; Hsia, K. J. Acta Mater. 2003, 51, 239-249.

(12) Huang, M.; Ritz, C. S.; Novakovic, B.; Yu, D.; Zhang, Y.; Flack, F.; Savage, D. E.; Evans, P. G.; Knezevic, I.; Liu, F.; Lagally, M. G. ACS Nano 2009, 3, 721-727.

(13) Guo, X.; Li, H.; Yeop Ahn, B.; Duoss, E. B.; Hsia, K. J.; Lewis, J. A.; Nuzzo, R. G. Proc. Natl. Acad. Sci. U.S.A. 2009, 106, $20149-20154$.

(14) Yu, D.; Liu, F. Nano Lett. 2007, 7, 3046-3050.

(15) Chun, I. S.; Verma, V. B.; Elarde, V. C.; Kim, S. W.; Zuo, J. M.; Coleman, J. J.; Li, X. J. Cryst. Growth 2008, 310, 2353-2358.

(16) Cottam, R. I.; Saunders, G. A.J. Phys. C: Solid State Phys. 1973, 6, 2105-2118.

(17) Prinz, V. Y.; Grutzmacher, D.; Beyer, A.; David, C.; Ketterer, B.; Deckardt, E. Nanotechnology 2001, 12, 399-402.

(18) Huang, M. H.; Boone, C.; Roberts, M.; Savage, D. E.; Lagally, M. G.; Shaji, N.; Qin, H.; Blick, R.; Nairn, J. A.; Liu, F. Adv. Mater. 2005, $17,2860$.

(19) Jain, S. C.; et al. Semicond. Sci. Technol. 1996, 11, 641.

(20) Paetzelt, H.; Gottschalch, V.; Bauer, J.; Herrnberger, H.; Wagner, G. Phys. Status Solidi A 2006, 203, 817-824.

(21) Chun, I. S.; Bassett, K.; Challa, A.; Li, X. Appl. Phys. Lett. 2010, 96, 251106

(22) Derickson, B. E. M.S. thesis, University of Illinois at UrbanaChampaign, 2010

(23) Kipp, T.; Welsch, H.; Strelow, C.; Heyn, C.; Heitmann, D. Phys. Rev. Lett. 2006, 96, 4 . 